\title{
Implication of Complex Vertebral Malformation and Bovine Leukocyte Adhesion Deficiency DNA-Based Testing on Disease Frequency in the Holstein Population
}

\author{
E. Schütz, M. Scharfenstein, and B. Brenig ${ }^{1}$ \\ Institute of Veterinary Medicine, Georg-August-University of Göttingen, Burckhardtweg 2, 37077 Göttingen, Germany
}

\begin{abstract}
Two inherited lethal disorders, bovine leukocyte adhesion deficiency (BLAD) and complex vertebral malformation (CVM), play a major role in breeding of Holstein cattle. Both inherited diseases are based on single nucleotide polymorphisms that have been known for 12 and $7 \mathrm{yr}$, respectively. A total of 25,753 cattle were genotyped for BLAD (18,200 tests) and CVM $(14,493$ tests) in our laboratory since the beginning of the genotyping programs for these diseases. Based on founder effects, the CVM mutation is thought to be linked to milk production. The BLAD was genotyped using RFLP until 2001; then a fluorescence resonance energy transfer assay on a LightCycler was used, as for CVM genotyping. By using single nucleotide polymorphism-aided breeding, the allelic frequency of the BLAD and CVM mutations in the active sire population was reduced from $9.4 \%$ in 1997 to $0.3 \%$ in 2007 (BLAD) and from $8.3 \%$ in 2002 to $2.3 \%$ in 2007 (CVM), with calculated half-life of the mutant allele of $2.1 \mathrm{yr}$ for BLAD and $3.6 \mathrm{yr}$ for CVM. An observed increase of BLAD frequency in 1999 could be attributed to the massive use of a BLAD-positive sire tested falsely negative in another laboratory. These data show that marker-assisted selection is capable of substantially reducing the frequency of a mutation within a period of not more than $5 \mathrm{yr}$. The different selection strategies against the lethal recessive allele in CVM and BLAD are reflected in the different reduction rates of the specific allele frequencies.

Key words: complex vertebral malformation, bovine leukocyte adhesion deficiency, lethal allele frequency, mutant allele half-life
\end{abstract}

\section{INTRODUCTION}

Currently, 40 disorders and traits in cattle have been characterized in which the causative mutation has been

Received March 5, 2008.

Accepted August 19, 2008.

${ }^{1}$ Corresponding author: bbrenig@gwdg.de identified at the DNA level (Online Mendelian Inheritance in Animals; http://omia.angis.org.au). However, the routine analysis of only a few of those has entered breeding programs so far and is in some instances mandatory for animals that are used for breeding. Two very important disorders in Holstein cattle that are routinely diagnosed are bovine leukocyte adhesion deficiency (BLAD) and complex vertebral malformation (CVM).

The molecular biological cause of BLAD was first described in 1992 and results from a missense mutation at amino acid position 383 in the ITGB2 gene ( $\beta 2$ integrin; GeneID: 281877) located on BTA1 between positions 131892340 and 131863180 (NC_007299) harboring 14 exons (Shuster et al., 1992a,b). The numbers refer to the current chromosomal location of the ITGB2 gene sequence in the bovine genome map (build 3.1; National Center for Biotechnology Information, 2008; http://www.ncbi.nlm.nih.gov). Affected cattle show a plethora of severe signs (e.g., ulcers of oral mucous membranes, gingivitis, loss of teeth, chronic pneumonia, impaired wound healing, anorexia, chronic diarrhea, and chronic dermatitis) that ultimately cause death (Nagahata, 2004). The introduction of the mutation into the Holstein population worldwide can be traced back to the very famous sire Osborndale Ivanhoe (US Holstein Herdbook registration number 1189870) who died in 1963 (Shuster et al., 1992b). Specimens with the BLAD allele have been identified in cattle starting in 1977 (Gilbert et al., 1993). Since a simple DNA-based diagnosis became available (Batt et al., 1994; Tammen et al., 1996), routine testing was introduced into the breeding programs, and the prevalence of the BLAD allele was significantly reduced. Recent reports on the BLAD allele frequency are available from Iran (Norouzy et al., 2005), China (Ma et al., 2006), Turkey (Akyuz and Ertugrul, 2006), India (Patel et al., 2007), Canada (Van Doormaal, 2008), and Poland (Czarnik et al., 2007; Rusc and Kaminski, 2007). In these studies the frequency of BLAD carriers is currently approximately 0.8 to $3.45 \%$ of the tested cattle.

The CVM is caused by a $\mathrm{G}>\mathrm{T} 559$ missense mutation in the SLC35A3 gene (solute carrier family 35 
member 3, UDP-N-acetylglucosamine transporter) on BTA3 (Thomsen et al., 2006). The SLC35A3 gene consists of 6 exons. Signs of CVM are malformations of the vertebral bones and ribs resulting in scoliosis and vertebral synostosis. Approximately 50\% of the affected cattle also show cardiac anomalies [e.g., interventricular septal defects, dextroposition of the aorta, right ventricular hypertrophy, and transposition of the aorta (Agerholm et al., 2001, 2004; Duncan et al., 2001; Revell, 2001)]. The CVM mutation was introduced into the Holstein population, most likely by the famous bull Penstate Ivanhoe Star (US Holstein Herdbook registration number 502041). Penstate Ivanhoe Star received the mutation from his mother, Penstate Lucifer Anna Star (US Holstein Herdbook registration number 3279562) because his father, Osborndale Ivanhoe, was CVM negative. In addition, Penstate Ivanhoe Star was a BLAD carrier, and therefore both defects were spread in the population through this famous sire. Early reports on CVM date back to 2000, and CVM has been described in several countries (Agerholm et al., 2001; Duncan et al., 2001; Nagahata et al., 2002; Berglund et al., 2004; Rusc and Kaminski, 2007; Ghanem et al., 2008). Because DNA-based testing of CVM became available only in 2002, studies on the current prevalence of CVM are still rare. In a report by Rusc and Kaminski (2007) 150 CVM carriers were identified out of 605 examined bulls in Polish Holstein cattle (24.8\%). In a study performed with 200 Japanese Holstein cows, 26 animals were heterozygote CVM carriers (13.0\%; Ghanem et al., 2008). Hence, in most Holstein populations the frequency of CVM carriers seems to be still high.

The current study reports the situation of the BLAD and CVM recessive lethal allele frequency in the German Holstein population including internationally used sires. Because several important Holstein sires are BLAD and CVM positive, the objective of this study was to verify whether the frequency of the lethal alleles for both disorders shows a similar reduction rate and whether a simple algorithm can describe the effects of marker-assisted selection on the recessive lethal alleles of CVM and BLAD.

\section{MATERIALS AND METHODS}

\section{Study Population}

During the study period from 1995 to 2007, a total of 32,693 genotype analyses were performed, of which 18,200 were BLAD and 14,493 were CVM. In 6,941 cases both genotypes were analyzed. The vast majority of tested cattle originated from Germany. Table 1
Table 1. Total number of tested cattle ${ }^{1}$

\begin{tabular}{|c|c|c|c|c|}
\hline \multirow[b]{2}{*}{ Year } & \multicolumn{2}{|c|}{ Sires } & \multicolumn{2}{|c|}{ Cows } \\
\hline & CVM & BLAD & CVM & BLAD \\
\hline 1995 & 5 & 761 & 3 & 191 \\
\hline 1996 & 22 & 1,129 & 1 & 227 \\
\hline 1997 & 25 & 1,469 & 1 & 358 \\
\hline 1998 & 67 & 1,528 & 5 & 282 \\
\hline 1999 & 64 & 1,291 & 8 & 205 \\
\hline 2000 & 55 & 1,254 & 9 & 220 \\
\hline 2001 & 274 & 1,211 & 57 & 293 \\
\hline 2002 & 2,948 & 1,037 & 869 & 216 \\
\hline 2003 & 1,921 & 1,243 & 463 & 199 \\
\hline 2004 & 2,000 & 1,313 & 390 & 158 \\
\hline 2005 & 1,743 & 1,208 & 332 & 161 \\
\hline 2006 & 1,505 & 1,092 & 162 & 72 \\
\hline 2007 & 1,375 & 985 & 189 & 97 \\
\hline$\Sigma$ & 12,004 & 15,521 & 2,489 & 2,679 \\
\hline
\end{tabular}

${ }^{1} \mathrm{CVM}=$ complex vertebral malformation; BLAD = bovine leukocyte adhesion deficiency.

gives an overview of tests performed during this period, stratified by year and sex.

The BLAD genotyping was done according to 2 different methods. Until 2001 a RFLP method was applied to 10,420 samples (Mirck et al., 1995; Mukhopadhyaya et al., 2000; Patel et al., 2007). From 2002 onward, this method was replaced by a fluorescence resonance energy transfer (FRET; Förster, 1946) assay (7,781 analyses; Lay and Wittwer, 1997). The CVM genotyping was started in 2001 first using a diagnostic sequencing technique, replaced by a FRET assay in 2002. Due to the greater error rate of diagnostic sequencing, all samples were reanalyzed with the FRET assay, and all results reported herein are based on the latter method.

\section{Breeding Policies for CVM and BLAD in the German Holstein Population}

According to the German Animal Protection Act $\S 11 b$, it is strictly forbidden to breed vertebrates where it can be expected that the offspring or further progeny will suffer from congenital defects that cause pain, disease, or injury. Therefore, the use of heterozygous parents carrying a recessive lethal allele, which could result in $25 \%$ of affected homozygous offspring, must be avoided. Consequently, the German Holstein Breeding Association had to implement routine DNA-based diagnosis of BLAD and CVM in the breeding programs immediately after the molecular cause was identified. Although a strict exclusion of all carriers would be advantageous to eliminate the mutated allele in almost one generation, this cannot be performed due to the fact that very important sires or cows might be affected. Hence, the general policy is to exclude carrier animals from the breeding program as far as possible and to 
cross cattle only if no homozygous affected offspring can be expected (e.g., heterozygous sires are crossed only to homozygous wild-type cows).

As of June 2008 not all sires have been tested for CVM because (i) they were born before the implementation of the DNA-based diagnosis, or (ii) they were not considered for breeding anymore. Currently, however, all sires that enter progeny testing are genotyped for CVM. The policies for the analysis of BLAD are different depending on the individual strategies of the breeding organizations. Some AI organizations genotype all of their test sires. Others genotype only those where the father or mother have been found to be heterozygous.

\section{DNA Isolation and Genotyping}

Whole blood was subjected to DNA extraction according to 2 different methods, a manual extraction method (Miller et al., 1988) or an automated silica-based method (MagnaPure, DNA-Isolation-Kit I - Blood fast protocol, Roche Applied Science, Mannheim, Germany). The purified DNA solution containing 20 to $100 \mathrm{ng} / \mu \mathrm{L}$ of DNA was used for all subsequent analyses.

For BLAD-RFLP, $1 \mu \mathrm{L}$ of DNA solution was subjected to 35 cycles of PCR in a total volume of $25 \mu \mathrm{L}$ containing $1 \mathrm{U}$ of Taq polymerase (various vendors), $2.5 \mu \mathrm{L}$ of $10 \times$ PCR buffer, $0.8 \mathrm{n} M$ dNTP, $2 \mathrm{~m} M \mathrm{MgCl}_{2}$, $1 \mu M$ of primer BLADfor-(5'-CCT GCA TCA TAT CCA CCA G-3'), and $1 \mu M$ BLADrev-(5'-GTT TCA GGG GAA GAT GGA G-3'). After PCR, $15 \mu \mathrm{L}$ of the resulting product was subjected to digestion with $20 \mathrm{U}$ of TaqI for $2 \mathrm{~h}$ at $65^{\circ} \mathrm{C}$, resulting in fragments of $152 / 191 \mathrm{bp}$ in the wild type and $343 \mathrm{bp}$ in the mutated variant. Fragments were analyzed with agarose gel electrophoresis (1.5\%) and visualized under UV light after staining with ethidium bromide.

For BLAD-FRET, $1 \mu \mathrm{L}$ of DNA solution was used in a total PCR volume of $20 \mu \mathrm{L}$ using the LightCycler Fast-Start Hybridization probes Kit (Roche Applied Science) with $2 \mathrm{mM} \mathrm{MgCl}$, containing $0.5 \mu M$ PCR primer BLADfor $0.5 \mu M$ BLADrev, $0.3 \mu M$ FRET anchor Probe BLADanchor (5'-FLOU-ACC TCT CCT ACT CCA TGG TGG A-P-3'), and $0.2 \mu M$ sensor probe BLADprobe (5'-ATC GAC CTG TAC TAC CGA T-ROX-3'). The resulting PCR reaction was transferred into LightCycler capillaries, placed into a LightCycler (model 1.2, Roche Applied Science) and subjected to 10 -min initial denaturation at $95^{\circ} \mathrm{C}, 38$ cycles of amplification with $4 \mathrm{~s}$ at $95^{\circ} \mathrm{C}, 10 \mathrm{~s}$ at $53^{\circ} \mathrm{C}$, and $10 \mathrm{~s}$ at $72^{\circ} \mathrm{C}$. After cycling the following melting analysis was performed: $30 \mathrm{~s}$ at $95^{\circ} \mathrm{C}, 90 \mathrm{~s}$ at $40^{\circ} \mathrm{C}$ (ramp rate $2^{\circ} \mathrm{C} / \mathrm{s}$ ), data collection 40 to $73^{\circ} \mathrm{C}$ with $0.2^{\circ} \mathrm{C} / \mathrm{s}$ transition rate. The melting points $(\mathbf{T m})$ are defined as maximum of the negative first derivative of the measured fluorescence over temperature. The Tm was $57^{\circ} \mathrm{C}$ for the wild-type and $48^{\circ} \mathrm{C}$ for the mutant variant, as recorded in channel $2(640 \mathrm{n} M)$. In each run a wild-type and a mutant control was included, and results were accepted if the deviation of Tm of unknowns from controls was less than $0.5^{\circ} \mathrm{C}$.

For CVM-FRET, exactly the same method as described for BLAD was applied using the following primers: $0.5 \mu M$ CVMfor $\left(5^{\prime}\right.$-GCC CTC AGA TTC TCA AGA G-3 ${ }^{\prime}$ ), $0.5 \mu M$ CVMrev (5'-GTT GAA TGT TTC TTA TCC ACA CT-3'), $0.3 \mu M$ anchor probe CVMrevWTA (5'-CY5.5-AGA CCT ACA AAT TGT GAG CCA GC-P-3'), and $0.2 \mu M$ sensor probe CVMrevWT (5'-ATG CTG TGA GAA CTG CCA-FLOU-3'). The $\mathrm{Tm}$, recorded in channel $3(705 \mathrm{~nm})$ were $61^{\circ} \mathrm{C}$ for the wild-type allele and $49^{\circ} \mathrm{C}$ for the mutant variant and were only accepted if not deviating more than $0.5^{\circ} \mathrm{C}$ from the in-run controls as described above. If both, CVM and BLAD were performed for one individual, the analysis was done in one capillary by adding both primer/probe sets to the reaction. In this case, dedicated fluorescein/ROX/CY5.5 color compensation was used.

For both assays the sensor probes were in-silico designed to maximize the $\Delta \mathrm{Tm}$ between the allelic variants (von Ahsen et al., 1999). The between-run Tm variability is less than $1.5^{\circ} \mathrm{C}$ and the $\Delta \mathrm{Tm}$ variability is negligible because they were lower than the technical resolution of the instrument. In turn, if a noticeable $\Delta$ Tm deviation in a sample occurs, such samples need to be re-run, and if confirmed a secondary technique should be applied (e.g., sequencing).

Allelic frequencies $(\mathrm{p}=$ wild-type; $\mathrm{q}=$ mutant $)$ were calculated based on the Hardy-Weinberg equation because homozygous mutated individuals undergo fetal death, are killed after birth based on the obvious phenotype (CVM), or die within the first months of life (BLAD). Therefore, for calculation of $\mathrm{p}$ and $\mathrm{q}$, the mutant homozygous frequencies were defined as

$$
\left(\frac{\sum p q}{2 \times \sqrt{\sum p p}}\right)^{2} .
$$

To assess the elimination of the mutant allele, data were stratified according to year of testing and an elimination equation was used to calculate the elimination constant with a best fit approach, minimizing the residual sum of squares.

$$
f_{q}(T)=f_{q}\left(T_{0}\right) \times e^{(-k T)},
$$


where $\mathrm{T}$ in years, $\mathrm{T}_{0}=$ first year of genotyping, and $\mathrm{k}$ $=$ elimination constant. For comparison purposes, the theoretical natural decline rate was calculated as follows under the assumption of random mating because as per Falconer and MacKay (1996) $\mathrm{q}^{2}$ can be considered lethal for population statistics (no $F_{1}$ contribution) for both BLAD and CVM:

$$
\frac{q}{1+q}
$$

This decline rate is per generation. To calculate the natural decline rate per year and compare it with the observed decline, an average generation of 5 yr was assumed.

\section{RESULTS}

In this study a total of 25,753 Holstein cattle were included (Table 1) of which 21,191 were sires and 4,562 were cows. Within the group of sires 12,004 tests for CVM and 15,521 for BLAD were performed. The number of tested sires per year corresponds to the number of sires selected to enter the German Holstein breeding program, typically at 15 to 18 mo of age. In the group of cows 2,489 tests for CVM and 2,679 for BLAD were performed. In Figure 1 the evolution of the frequency of the mutated alleles (q) of CVM and BLAD in the cow cohort is shown for the group of tested cows. In the first 2 yr only 194 cows were tested due to the delayed implementation of the official regulations. The maximum frequency of BLAD was reached in 2000 with $24 \%$ and by 2007 had declined to approximately 1.6\%. The frequency of the mutated CVM allele in cows shows a different evolution. The overall frequency remained lower compared with BLAD ranging from 9 to $16 \%$. However, the frequency did not decline over the period of time. The high frequencies in the tested cow group reflect the fact that they are a highly selected sample, of relatively small size, corresponding primarily to bull dams that are thought to have a high likelihood of carrying the mutant allele. Therefore, these frequencies may not reflect the frequencies in the overall cow population or their evolution over time. In the group of tested males, the situation is different in the male cohort for the 2 disorders (Figure 2). In the first $2 \mathrm{yr}$ of BLAD-genotyping, in 1995 and 1996, a total of 1,890 sires were tested, with an allele frequency of $2.0 \%$. During this period, a few sires commonly used in Germany were identified as carriers of the mutation, leading to a more selective use of the test in later years for cattle with pedigrees indicating a higher risk of being mutation carriers. From 1997 to 2007 the number of

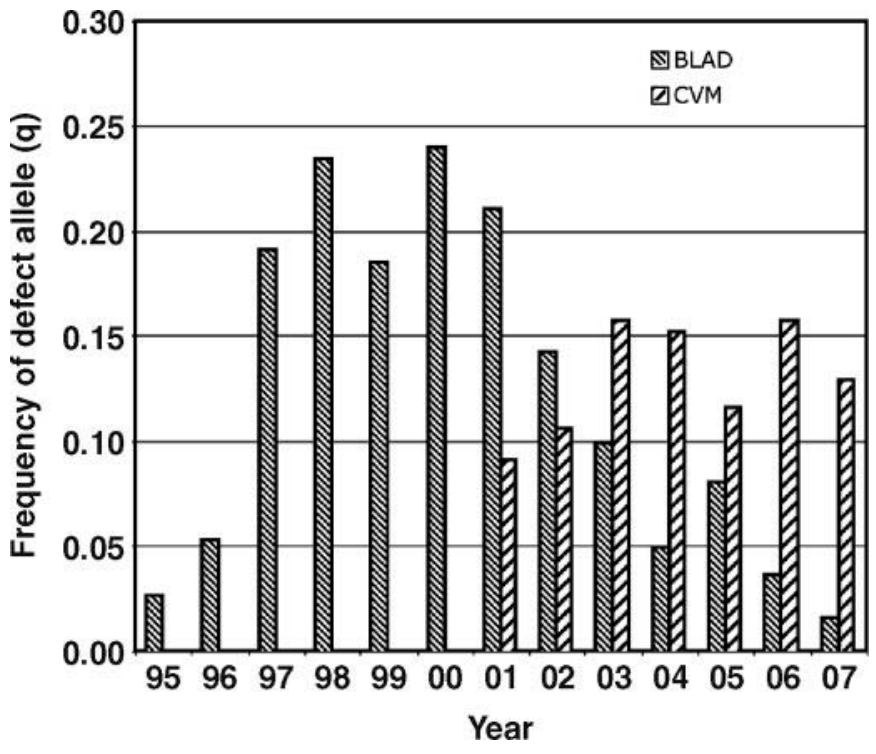

Figure 1. Frequency of mutant allele for bovine leukocyte adhesion deficiency (BLAD) and complex vertebral malformation (CVM) in German Holstein cows.

BLAD tests in sires at risk ranged from 985 to 1,528 per year. The allelic frequency of the mutant variant in 1997 was $9.4 \%$, a highly significant increase compared with previous years $\left(\chi^{2}: P<1 \times 10^{-5}\right)$. After 1997, the mutation frequency decreased with an estimated half-life of $3.0 \mathrm{yr}\left(\mathrm{r}^{2}=0.88\right)$, as shown in Figure 2A. However, in 2000 one sire was found to carry the mutation, which was unexpected by pedigree analysis. It was shown that one ancestor had been mistyped abroad, which led to a redefinition of the risk group in the German Holstein breed. Meanwhile, the carrier bull was highly used, which led to a significant increase of the allelic frequency of the mutant from $3.1 \%$ in 1999 to $5.4 \%$ in $2001\left(\chi^{2}: 1999-2000, P=0.25 ; 2000-2001, P\right.$ $<0.01)$. The evolution of variant frequency from that time point on shows the effect of breeding based on BLAD genotyping. After 2001, the frequency of the BLAD mutation decreased with an estimate half-life of $2.1 \mathrm{yr}\left(\mathrm{r}^{2}=0.94\right)$ reaching a frequency of $0.3 \%$ at the end of 2007, which is significantly faster compared with the natural decline or the decline rate resulting from avoiding heterozygous mating.

The elimination rate of the CVM mutation is less pronounced than for BLAD, with a calculated half-life of $3.6 \mathrm{yr}\left(\mathrm{r}^{2}=0.96\right.$; Figure $\left.2 \mathrm{~B}\right)$. In contrast to BLAD, a homozygous mutation in CVM is easily diagnosed because of the obvious phenotype of the affected calf. Therefore, many sires suspected of being carriers were already known and the risk group was already defined.

In addition, the use of only homozygous negative animals in the breeding process was less stringent for 
CVM than for BLAD. However, the elimination rate of both BLAD and CVM was considerably faster than would have occurred through natural selection.

\section{DISCUSSION}

In looking at over a decade of genotyping for BLAD and CVM in the Holstein breed, it can be seen that different selection strategies define the decline rate of the mutation. A lethal recessive allele will normally be eliminated at the rate shown in equation [3], given that homozygous recessives cannot mate (Falconer and MacKay, 1996). This process alone is extremely inefficient for the elimination of a rare allele from a population (see Figure 2). Therefore breeding programs are necessary to reduce $q$ in a reasonable time. If a DNA-based test is available to detect heterozygotes, a more efficient method to eliminate $q$ is the testing of sires and exclusion of heterozygotes (Rønningen, 1973). Using such a strategy would eliminate any $q q$ individuals in the following generation, and the allele frequency would be halved in each generation. However, such strategies are influenced by the fact that several genes may have direct or indirect effects or are in linkage disequilibrium with economically important traits. In this case the prediction of the allele frequency is more complicated.

The data shown in this study reflect the annual changes in allelic frequencies. Only those sires that are newly selected for breeding each year are genotyped (typically at an age of about 15 to $18 \mathrm{mo}$ ). Furthermore, cattle that are expected to be homozygous $p p$ by pedigree are not necessarily tested. Therefore these data should not serve as a population overview. Nevertheless, the data reflect a large proportion of all sires that are preselected each year for breeding and could be used as parents.

For BLAD, which is not considered to be linked to any economically important trait in dairy cows, a very rapid elimination of the mutation was achieved by a rigid selection strategy, where carriers were not used for breeding at all. As shown in Figure 1 and 2, the decline of the mutant BLAD allele is similar in sires and cows. The fact that the elimination rate is about $45 \%$ per year shows that a certain proportion of ancestors (mainly cows) have an unknown BLAD genotype.

In contrast, the situation for CVM is complicated by the fact that the mutation is linked to milk quality and quantity, which is a major economic factor in dairy cows. This is most probably due to a founder effect resulting from Osborndale Ivanhoe, which was heavily used, due to its good performance, before the sire was proven to be a CVM carrier.
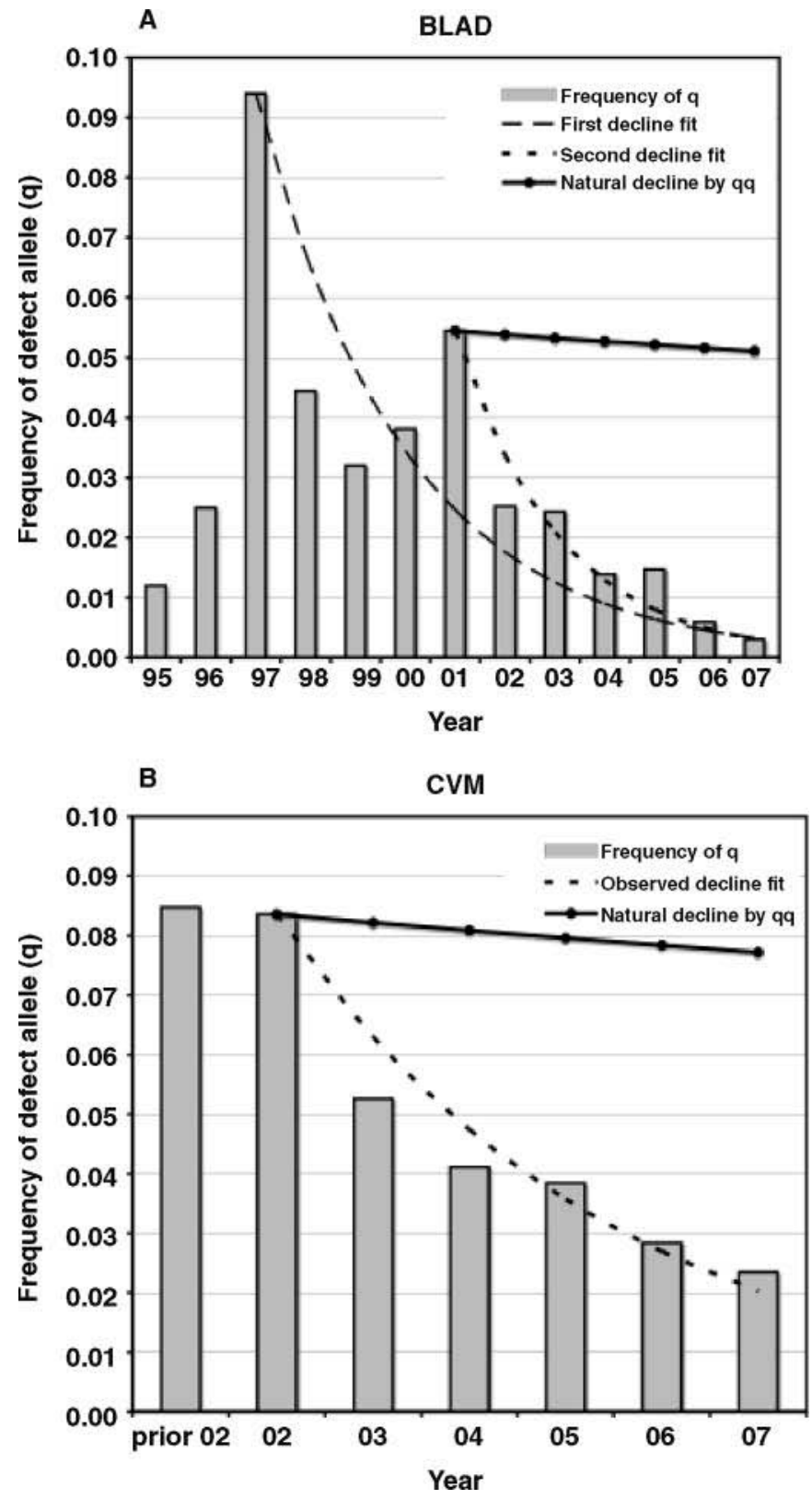

Figure 2. Frequency and statistics of mutant allele for bovine leukocyte adhesion deficiency (BLAD; panel A) and complex vertebral malformation (CVM; panel B) in Holstein sires. The actual elimination rates calculated as best fit to natural elimination formula. Theoretical decline rates for natural selection (qq) under the assumption of random mating are given. Calculations are given in the Materials and Methods section.

The breeding efforts to eliminate the lethal recessive allele are a compromise between the aim to eliminate the inherited disease and economic considerations. Therefore, the above-mentioned founder effect has led to the tolerance of heterozygous mutant carriers, slowing down the elimination. In conclusion, this retrospective study shows the effect of marker-assisted selection on 
the eradication of 2 inherited diseases in a production population.

\section{REFERENCES}

Agerholm, J. S., C. Bendixen, O. Andersen, and J. Arnbjerg. 2001. Complex vertebral malformation in Holstein calves. J. Vet. Diagn. Invest. 13:283-289.

Agerholm, J. S., C. Bendixen, J. Arnbjerg, and O. Andersen. 2004. Morphological variation of "complex vertebral malformation" in Holstein calves. J. Vet. Diagn. Invest. 16:548-553.

Akyuz, B., and O. Ertugrul. 2006. Detection of bovine leukocyte adhesion deficiency (BLAD) in Turkish native and Holstein cattle. Acta Vet. Hung. 54:173-178.

Batt, C. A., P. Wagner, M. Wiedmann, J. Luo, and R. Gilbert. 1994. Detection of bovine leukocyte adhesion deficiency by nonisotopic ligase chain reaction. Anim. Genet. 25:95-98.

Berglund, B., A. Persson, and H. Stalhammar. 2004. Effects of complex vertebral malformation on fertility in Swedish Holstein cattle. Acta Vet. Scand. 45:161-165.

Czarnik, U., G. Grzybowski, S. Kaminski, B. Prusak, and T. Zabolewicz. 2007. Effectiveness of a program aimed at the elimination of BLAD-carrier bulls from Polish Holstein-Friesian cattle. J. Appl. Genet. 48:375-377.

Duncan, R. B. Jr, C. B. Carrig, J. S. Agerholm, and C. Bendixen. 2001. Complex vertebral malformation in a Holstein calf: Report of a case in the USA. J. Vet. Diagn. Invest. 13:333-336.

Falconer, D. S., and T. F. C. MacKay. 1996. Introduction to Quantitative Genetics. 4th ed. Longman Green, Essex, UK.

Förster, T. 1946. Energiewanderung und Fluoreszenz. Naturwissenschaften 6:166-175.

Ghanem, M. E., M. Akita, T. Suzuki, A. Kasuga, and M. Nishibori. 2008. Complex vertebral malformation in Holstein cows in Japan and its inheritance to crossbred $\mathrm{F}_{1}$ generation. Anim. Reprod. Sci. 103:348-354.

Gilbert, R. O., W. C. Rebhun, C. A. Kim, M. E. Kehrli Jr, D. E. Shuster, and M. R. Ackermann. 1993. Clinical manifestations of leukocyte adhesion deficiency in cattle: 14 cases (1977-1991). J. Am. Vet. Med. Assoc. 202:445-449.

Lay, M. J., and C. T. Wittwer. 1997. Real-time fluorescence genotyping of factor V Leiden during rapid-cycle PCR. Clin. Chem. 43:22622267.

Ma, J. Z., Y. D. Cui, Z. B. Zhu, H. W. Cao, and F. Z. Piao. 2006. Investigation on bovine leukocyte adhesion deficiency. Yi Chuan 28:1233-1236.

Miller, S. A., D. D. Dykes, and H. F. Polesky. 1988. A simple salting out procedure for extracting DNA from human nucleated cells. Nucleic Acids Res. 16:1215.

Mirck, M. H., T. Von Bannisseht-Wijsmuller, W. J. TimmermansBesselink, J. H. Van Luijk, J. B. Buntjer, and J. A. Lenstra. 1995. Optimization of the PCR test for the mutation causing bovine leukocyte adhesion deficiency. Cell Mol. Biol. (Noisy-le-grand) 41:695-698.

Mukhopadhyaya, P. N., H. H. Mehta, and R. N. Rathod. 2000. A method for the simulation of normal, carrier and affected controls for PCR-RFLP screening of a genetic disease in dairy cattle. Mol Cell. Probes 14:381-384.

Nagahata, H. 2004. Bovine leukocyte adhesion deficiency (BLAD): A review. J. Vet. Med. Sci. 66:1475-1482.

Nagahata, H., H. Oota, A. Nitanai, S. Oikawa, H. Higuchi, T. Nakade, T. Kurosawa, M. Morita, and H. Ogawa. 2002. Complex vertebral malformation in a stillborn Holstein calf in Japan. J. Vet. Med. Sci. 64:1107-1112.

Norouzy, A., M. R. Nassiry, F. Eftekhari Shahrody, A. Javadmanesh, M. R. Mohammad Abadi, and G. E. Sulimova. 2005. Identification of bovine leucocyte adhesion deficiency (BLAD) carriers in Holstein and Brown Swiss AI bulls in Iran. Genetika 41:1697-1701.

Patel, R. K., K. M. Singh, K. J. Soni, J. B. Chauhan, and K. R. Sambasiva Rao. 2007. Low incidence of bovine leukocyte adhesion deficiency (BLAD) carriers in Indian cattle and buffalo breeds. J. Appl. Genet. 48:153-155.

Revell, S. 2001. Complex vertebral malformation in a Holstein calf in the UK. Vet. Rec. 149:659-660.

Rønningen, K. 1973. Change in the frequency of recessive gene due to selection of males in artificial insemination. Acta Agric. Scand. $23: 157-164$.

Rusc, A., and S. Kaminski. 2007. Prevalence of complex vertebral malformation carriers among Polish Holstein-Friesian bulls. J. Appl. Genet. 48:247-252.

Shuster, D. E., B. T. Bosworth, and M. E. Kehrli Jr. 1992a. Sequence of the bovine CD18-encoding cDNA: Comparison with the human and murine glycoproteins. Gene 114:267-271.

Shuster, D. E., M. E. Kehrli Jr., M. R. Ackermann, and R. O. Gilbert 1992b. Identification and prevalence of a genetic defect that causes leukocyte adhesion deficiency in Holstein cattle. Proc. Natl. Acad. Sci. USA 89:9225-9229.

Tammen, I., H. Klippert, A. Kuczka, A. Treviranus, J. Pohlenz, M. Stober, D. Simon, and B. Harlizius. 1996. An improved DNA test for bovine leucocyte adhesion deficiency. Res. Vet. Sci. 60:218 221

Thomsen, B., P. Horn, F. Panitz, E. Bendixen, A. H. Petersen, L. E. Holm, V. H. Nielsen, J. S. Agerholm, J. Arnbjerg, and C. Bendixen. 2006. A missense mutation in the bovine SLC35A3 gene, encoding a UDP-N-acetylglucosamine transporter, causes complex vertebral malformation. Genome Res. 16:97-105.

Van Doormaal, B. 2008. Understanding genetic recessives in Holsteins. Canadian Dairy Network http://www.cdn.ca/articles.php June 2008. Accessed Oct. 21, 2008.

von Ahsen, N., M. Oellerich, V. W. Armstrong, and E. Schütz. 1999. Application of a thermodynamic nearest-neighbor model to estimate nucleic acid stability and optimize probe design: Prediction of melting points of multiple mutations of apolipoprotein B-3500 and factor $\mathrm{V}$ with a hybridization probe genotyping assay on the LightCycler. Clin. Chem. 45:2094-2101. 